\title{
Accelerometer-based Energy Expenditure Estimation Methods and Performance Comparison
}

\author{
Fang-Chen Chuang \\ Dept. of Electrical Engineering \\ National Cheng Kung University \\ Tainan, Taiwan \\ nono701107@yahoo.com.tw
}

\author{
Ya-Ting C. Yang \\ Institute of Education \\ National Cheng Kung University \\ Tainan, Taiwan \\ yangyt@mail.ncku.edu.tw
}

\author{
Jeen-Shing Wang \\ Dept. of Electrical Engineering \\ National Cheng Kung University \\ Tainan, Taiwan \\ jeenshin@mail.ncku.edu.tw
}

\begin{abstract}
This paper presents two accelerometer-based metabolic equivalent (MET) estimation methods: 1) MET regression models estimation; and 2) a mono-exponential MET estimation equation. The MET estimation methods can predict MET for the following seven categories of activities: sitting, standing, walking upstairs, walking downstairs, level walking, running, and cycling. The intensity of these activities ranged from sedentary ( $\leq 1.5$ METs) to vigorous ( $>6$ METs), where 1 MET is equivalent to the metabolic rate associated with sitting. First, MET regression model estimations were developed, including a single MET regression model and multiple MET regression models. Then, the second estimation method, a mono-exponential MET estimation equation, was developed to enhance estimation accuracy in non-steady and steady states during exercise. The experimental results successfully validate the effectiveness of the proposed method, the mono-exponential MET estimation equation, which substantially ameliorates estimation errors in non-steady states and achieves satisfactory accuracy for both non-steady and steady states.
\end{abstract}

Keywords-accelerometer, energy expenditure, metabolic equivalent of task, regression model estimation, non-steady state.

\section{INTRODUCTION}

Along with advances in science and technology, changes in daily lifestyles are resulting in the increased prevalence of physical inactivity. This lack of physical activity not only increases the incidence of chronic illnesses causing substantial financial and medical burdens on governments, but also seriously affects living quality. Previous studies [1], [2] have suggested the importance of physical activity in disease prevention. Existing tools for evaluating physical activity and EE include activity questionnaires, indirect calorimeters, doubly labeled water technique, electrocardiographs and accelerometers [3]. Due to the advantages of small size, portability, low power consumption, and low cost, accelerometers have become popular tools for objective measurement of physical activity and estimation of EE.

The EE estimation method utilizes features generated from both acceleration signals and personal parameters (height and weight) to construct a single linear regression model [4], [5]-[8]. However, using a single linear regression model to estimate EE cannot obtain satisfactory accuracy when physical activity intensity levels range from sedentary to vigorous. For example, Crouter et al. [4] compared the accuracy of different MET regression models developed by Actigraph, Actical, and AMP-331 accelerometers. Participants wore the three devices on their waists simultaneously. The experimental results showed that Actigraph and Actical overestimated the energy cost of walking and static activity, while seriously underestimating vigorous-intensity activities. AMP-331 provided a closer estimation of the energy cost during walking but slightly overestimated sedentary- and light-intensity activities while underestimating vigorous-intensity activities. In order to improve the accuracy of EE estimation, multiple regression models have been developed. For instance, Crouter et al. [9] asked participants to wear a uniaxial Actical accelerometer on their waists. When the count was $\leq 10$, the estimated MET was set as 1 . Two regression models were developed for counts $>10$. When count was $>10$ and the coefficient of variation $(\mathrm{CV})$ was $\leq 13$, an exponential regression model was used to estimate $\mathrm{EE}$ for walking and running activities, resulting in a coefficient of determination $\left(\mathrm{R}^{2}\right)$ of 0.912 and standard error of the estimation (SEE) of 0.149 METs. Alternatively, when the $\mathrm{CV}$ was $>13$, a regression model for lifestyle/leisure time physical activity was constructed, resulting in an $R^{2}$ of 0.884 and SEE of 0.804 METs.

In this paper, a wearable activity sensor system and a mono-exponential MET estimation equation were proposed for long-term MET estimation. The advantages of the wearable activity sensor system and the proposed estimation method, named "the mono-exponential MET estimation equation", include: 1) the system can detect and record the motion accelerations of various physical activities ranging from sedentary to vigorous intensity levels for long-term monitoring; and 2) the proposed MET estimation method substantially reduces estimation errors for the non-steady state and achieves satisfactory accuracy for both non-steady and steady states.

The remainder of this paper is organized as follows. In Section II, we introduce the experimental protocol and hardware devices in detail. The different MET estimation methods, including MET regression model estimation and a mono-exponential MET estimation equation are presented in Section III. Then, in Section IV, the experimental results are presented to validate the effectiveness of the proposed approach. Conclusions are provided in the final section. 


\section{EXPERIMENTAL PROTOCOL}

\section{A. Hardware Devices}

\section{- Wearable Activity Sensor System}

Each sensor module consists of a triaxial accelerometer (MMA7445L, Freescale ${ }^{\circledR}$ ), an MCU module (Microchip PIC24FJ64GA002), a wireless communication module (Nordic nRF2401+ and Bluetooth), and a storage module (16 MB flash memory). In this study, the accelerometer's sensitivity was set from $-8 \mathrm{~g}$ to $+8 \mathrm{~g}$. The Microchip ${ }^{\circledR}$ PIC24FJ64GA002 was selected as the MCU module and was responsible for the following tasks: 1) timing flow control for regularly retrieving accelerometer data from the sensor module via an $\mathrm{I}^{2} \mathrm{C}$ bus, 2) converting analog acceleration signals into digital signals via its embedded A/D converter, 3 ) wireless communication control and module synchronization, and 4) peripheral component control. The wireless communication module includes a Nordic ${ }^{\circledR}$ nRF24L01+ wireless RF transceiver and a BTM401 Bluetooth ${ }^{\circledR}$ module. The Nordic ${ }^{\circledR}$ nRF24L01+ wireless RF transceiver is used to execute timing synchronization and data transmission among sensor modules worn on different positions of a human body. The BTM401 Bluetooth ${ }^{\circledR}$ module serves as the standard communication channel between the host of the body sensor network and standardized devices such as PCs or smart phones. The storage module employed in the sensor module is an MXIC $\AA$ MX25L128 flash memory with a $16 \mathrm{MB}$ storage capacity. A Microchip ${ }^{\circledR}$ PIC24FJ64GA002 main controller is selected to maximize the performance and minimize the power consumption of the sensor module. The sampling rate $(f s)$ of the sensor module is $30 \mathrm{~Hz}$ with 12-bit data resolution, while power consumption is $12.2 \mathrm{~mA}$ at 4.2 V.

\section{- Portable Indirect Calorimeter System}

An indirect calorimeter system (Cosmed $\mathrm{K} 4 \mathrm{~b}^{2}$ ) was used to collect oxygen consumption in this experiment. According to the manufacturer's guidelines, the Cosmed $\mathrm{K} 4 \mathrm{~b}^{2}$ oxygen analyzer and carbon dioxide analyzer were calibrated before the experiment. The system weighs $1.5 \mathrm{~kg}$, including the battery and a specially designed harness, and is worn on participants' chest by a chest harness. A flexible face mask was placed over each participant's mouth and nose using a nylon mesh hairnet secured by Velcro straps. This mask was attached to a flowmeter (bidirectional digital turbine) and an optoelectronic reader. A disposable gel seal was placed between the participant and the face mask in order to prevent air leaks from the face mask. Finally, each participant's physical characteristics (age, height, weight, and gender) were entered into the Cosmed $\mathrm{K} 4 \mathrm{~b}^{2}$. During the experiment, participants wore the calorimeter system and the wearable activity sensor system to collect oxygen consumption and acceleration signals, which were recorded in the memory of the Cosmed $\mathrm{K} \mathrm{b}^{2}$ and then downloaded to a PC for analysis.

\section{B. Participants}

Ten participants, aged 20 to 25 years old, with an average body mass index (BMI) of $21.78 \pm 2.82 \mathrm{~kg} / \mathrm{m}^{2}$ were recruited to participate in this experiment. All participants were nonsmokers, disease-free, and not currently taking medications known to influence metabolic rats.

\section{Experimental Procedures}

All participants were asked to complete eleven activities, including various lifestyle activities and exercises, from seven categories: 1) Sitting: doing computer work and wiping the ground with a dry cloth; 2) Standing: sweeping, washing dishes, cleaning tables, and picking up objects; 3 ) Upstairs: walking upstairs; 4) Downstairs: walking downstairs; 5) Walking at a normal pace on a sports field; 6) Running on a sports field; 7) Riding a bicycle on a sports field. Before this experiment, each participant was instructed to wear the portable indirect calorimeter system and the activity sensor system. Participants attached the calorimeter system to their chests. The activity sensor system was comprised of two sensor modules mounted on participants' wrists and ankles. Participants performed each activity for 3 minutes, and took a rest between activities to ensure that their heart rates were below $100 \mathrm{bpm}$. Acceleration signals and oxygen consumption $\left(\dot{V}_{2}\right)$ during the 3 minutes were extracted for analysis. Acceleration data and oxygen consumption $\left(\mathrm{VO}_{2}\right)$ were segmented into non-overlapping windows of $1 \mathrm{~min}$ in length. Important features were extracted from the windowed acceleration data for physical classification and multiple MET regression model construction. $\dot{V O} \mathrm{O}_{2}(\mathrm{ml} / \mathrm{min})$ was averaged over a 1 -min period and converted to $\dot{V} O_{2}\left(\mathrm{ml} \cdot \mathrm{kg}^{-1} \cdot \mathrm{min}^{-1}\right)$ which divides by 3.5 to obtain the actual MET for multiple MET regression model construction. 1 MET is defined as (1).

$$
1 \mathrm{MET}=3.5 \mathrm{ml} / \mathrm{min} / \mathrm{kg}=1 \mathrm{kcal} / \mathrm{hr} / \mathrm{kg},
$$

\section{MET Estimation Methods}

In this paper, an MET regression model estimation and our proposed estimation method, a mono-exponential MET estimation equation, are evaluated. MET regression model estimations, including a single MET regression model and multiple MET regression models, are introduced in detail in Section A. The mono-exponential MET estimation equation introduced in Section B was developed to enhance the estimation accuracy during non-steady state exercise.

\section{A. MET Regression Model Estimation}

MET regression model estimation consists of three steps: signal pre-processing, feature generation, and MET regression model construction. In this study, the purpose of MET regression model construction is to construct single and multiple MET regression models for estimating MET for different intensity levels of physical activities.

\section{- $\quad$ Signal Pre-processing}

Signal pre-processing is a required procedure for analyzing signals collected by the sensor system. During activities/movement, the sensor system records accelerations including both gravitational acceleration (GA) and body acceleration (BA). Therefore, a high-pass filter is required to remove gravitational acceleration and to obtain the BA component after calibration. In general, it is difficult to 
analyze and recognize activities from a long-term and continuous sequence of acceleration data. During data preprocessing, accelerations are segmented into nonoverlapping windows of 1 min in length (1800 sample points) and signal vector magnitude (SVM) is calculated for further analysis, as described in the following section.

$$
\operatorname{SVM}(n)=\sqrt{\operatorname{Acc}_{x_{n}}{ }^{2}+A \operatorname{Ac}_{y_{n}}{ }^{2}+A \mathrm{Ac}_{z_{n}}{ }^{2}}
$$

Where $A \operatorname{cc}_{x_{n}}, A \operatorname{cc}_{\mathrm{y}_{n}}$, and $A \mathrm{cc}_{z_{n}}$ are the $n$th sample of the $x$-, $y$-, and $z$-axis acceleration signals generated from the sensor activity module.

\section{- Feature Generation}

Characteristics of acceleration signals from different physical activities were obtained by extracting features from pre-processed SVM signals, and included thirteen generated features: 1) eleven time-domain features: count, standard deviation (STD), interquartile range (IQR), mean absolute deviation (MAD), norm, ratio of stance phase (RSP), skewness, kurtosis, the first, second, and third quartile (Q1, Q2, and Q3), and 2) two frequency-domain features: energy and entropy. A total of 25 features were generated from the feature generation process. The features are used to construct various MET regression models for seven activity categories to estimate MET, respectively.

\section{- MET Regression Model Construction}

A flowchart for MET regression model construction, comprising acceleration acquisition, signal pre-processing, feature generation, bivariate correlation analysis, and MET regression model construction by stepwise regression. The procedures for signal pre-processing and feature generation were described in the above sections. The generated features were imported into SPSS ${ }$ 17.0, which is used to perform bivariate correlation analysis. Features with the high correlation $(p<0.05)$ were selected as input variables for constructing single/multiple MET regression models by a SPSS ${ }$ Statistics 17.0 regression function called "stepwise regression". Table I lists single/multiple MET regression models for seven activity categories. In this study, the single regression model and multiple MET regression models were referred to as "Method (1)" and "Method (2)", respectively.

TABLE I. MET REgRESSION MODELS

\begin{tabular}{|c|c|c|}
\hline $\begin{array}{l}\text { Type of } \\
\text { Model }\end{array}$ & Activity & MET Regression Models \\
\hline $\begin{array}{c}\text { Single } \\
\text { Model } \\
\text { (Method 1) }\end{array}$ & All & $\begin{array}{l}M E T_{e}=7.916 \times S T D_{h}-0.918 \times R A S-0.102 \\
\times B M I+33.244 \times p 25_{a}+11.867 \times p 50_{a}- \\
17.002 \times \text { Mean }_{a}+4.474 \times p 75_{h}-3.410 \times p 50_{h} \\
-1.984 \times I Q R_{h}+4.656\end{array}$ \\
\hline \multirow{5}{*}{$\begin{array}{l}\text { Multiple } \\
\text { Models } \\
\text { (Method 2) }\end{array}$} & Sit & $\begin{aligned} M E T_{e} & =0.0297 \times \text { Energy }_{a}+0.0518 \times \text { Count }_{h} \\
& +0.2665 \times \text { Count }_{a}-2.0219\end{aligned}$ \\
\hline & Stand & $\begin{aligned} M^{M E T_{e}}= & 0.1827 \times \text { Entropy }_{a}-0.3785 \times B M I \\
& +0.0924 \times \text { Weight }^{2} 0.038 \times \text { Count }_{h} \\
& -3.8012 \times \text { RAS }_{a}+6.4213\end{aligned}$ \\
\hline & Walk & $M E T_{e}=0.009 \times E \operatorname{nergy}_{a}+1.208$ \\
\hline & Run & $M E T_{e}=0.0051 \times E \operatorname{nergy}_{h}+0.0063 \times E \operatorname{nergy}_{a}+3.0203$ \\
\hline & Cycling & $M E T_{e}=12.5001 \times V a r_{h}+2.6019$ \\
\hline
\end{tabular}

\begin{tabular}{|c|c|c|}
\hline $\begin{array}{c}\text { Type of } \\
\text { Model }\end{array}$ & Activity & MET Regression Models \\
\hline \multirow{3}{*}{} & Upstairs & $M E T_{e}=10.6140 \times I Q R_{h}+0.0089 \times E$ nergy $_{a}-0.2778$ \\
\cline { 2 - 3 } & $\begin{array}{c}\text { Downstair } \\
\mathrm{S}\end{array}$ & $M E T_{e}=0.2507 \times$ Skewness $_{a}+1.5624$ \\
\hline
\end{tabular}

\section{B. A Mono-exponential MET Estimation Equation}

The body does not always achieve a steady state and is more often kept in a non-steady state during exercise of moderate- or vigorous-intensity. During moderate- or vigorous-intensity exercise, oxygen uptake or metabolism can be evaluated in two ways: 1) static estimation for the steady state condition, and 2) dynamic response during the beginning and the end of exercise (the non-steady state condition). From Fig. 1, it can be seen that MET consumption at the beginning of running exercise remains in a non-steady state. After 1 minute of remaining in a nonsteady state (from the beginning), a steady state is reached. Finally, when the participant stops running, MET consumption decreases from the stopping point.

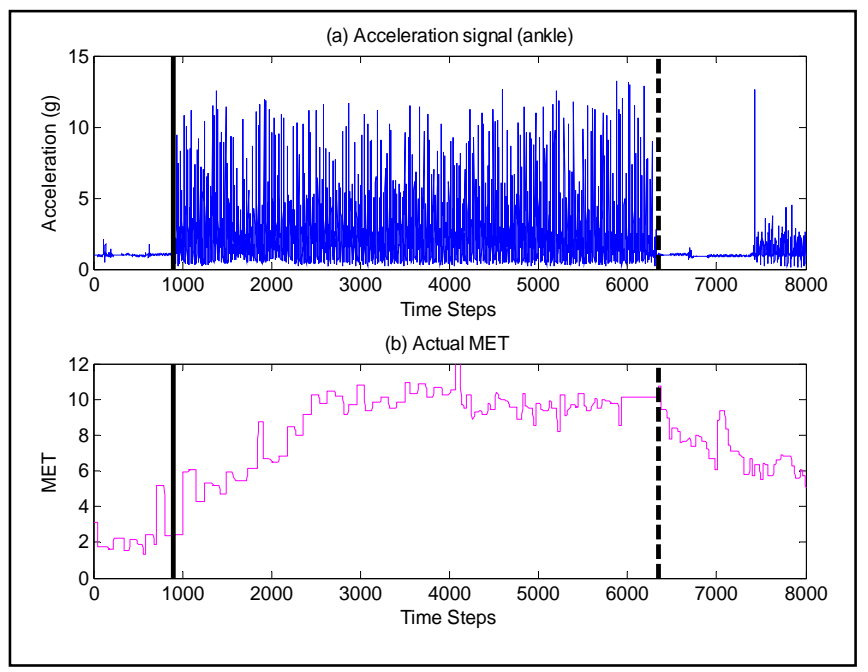

Figure 1. Acceleration signals generated from the ankle sensor and the actual METs measured by the $\mathrm{K} 4 \mathrm{~b}^{2}$ during running. The solid line represents the starting point, and the dashed line represents the stopping point.

However, the drawback of single/multiple MET regression model estimation is that the estimation results from the non-steady state, during moderate- and vigorousintensity activities, are unsatisfactory. In order to improve the accuracy of MET estimation for the non-steady state, an MET estimation method, a mono-exponential MET estimation equation, was used. This method is also referred to as "Method (3)". The mono-exponential equation can be expressed as follows.

$$
M E T_{\text {out }}(\mathrm{t}+1)=M E T_{\text {in }}(\mathrm{t})-M E T_{\text {in }}(\mathrm{t}) \times e^{-\frac{1}{p_{\text {up }}}}+M E T_{\text {out }}(\mathrm{t}) \times e^{-\frac{1}{p_{\text {down }}}},
$$

where $\mathrm{MET}_{\text {out }}$ is the output, and $\operatorname{MET}_{\text {out }}(0)$ is set as 0 . $\mathrm{MET}_{\text {in }}$ is the input generated from multiple MET regression models. $p_{\text {up }}$ and $p_{\text {down }}$ are the coefficients of the monoexponential equation. 


\section{EXPERIMENTAL RESULTS}

\section{A. Results of MET Regression Model Estimation}

In this study, performance evaluation was based on the standard error of the estimate (SEE) and the coefficient of determination $\left(R^{2}\right)$ between the desired MET and the estimated MET. The sum of squares due to error (SSE) and total sum of squares (SSTO) are represented as follows:

$$
\begin{aligned}
& S S E=\sum_{t=1}^{n}\left(\mathrm{MET}_{a}-\mathrm{MET}_{e}\right)^{2} \\
& S S E=\sum_{t=1}^{n}\left(\mathrm{MET}_{a}-\overline{\mathrm{MET}_{a}}\right)^{2} \\
& S S E=\sqrt{\frac{S S E}{n-p-1}}
\end{aligned}
$$

where $n$ is the total amount of testing data. $M E T_{a}$ and $M E T_{e}$ represent actual MET and estimated MET. $\overline{\mathrm{MET}_{a}}$ is the mean of $M E T_{a} . p$ denotes the number of features used in the regression model. For example, $p$ is equal to 9 for the single MET regression model. For the multiple MET regression models, the $p$ of "sitting", "standing", "level walking", "running", "cycling", "walking upstairs", and "walking downstairs" were equal to $3,5,1,2,1,2$, and 1, respectively. Table II shows the SEE and $\mathrm{R}^{2}$ for the single/multiple regression models. The mean was 0.702 and the STD of residual errors was 1.215 METs for the single MET regression model. For the multiple regression models, the mean was 0.613 and the STD was 0.709 METs. These results indicate that the performance of the multiple MET regression models was better than the single MET regression model. Overall, the performance of MET estimation generated by the multiple regression models outperformed that generated by the single regression model.

TABLE II. RESULTS FOR MET REGRESSION MODELS

\begin{tabular}{|c|c|c|c|}
\hline $\begin{array}{c}\text { Type of } \\
\text { Model }\end{array}$ & Activity & SEE & $\mathbf{R}^{\mathbf{2}}$ \\
\hline Single Model & All & 1.438 & 0.815 \\
\hline \multirow{4}{*}{$\begin{array}{c}\text { Multiple } \\
\text { Models }\end{array}$} & Sitting & 0.473 & 0.821 \\
\cline { 2 - 4 } & Standing & 0.354 & 0.704 \\
\cline { 2 - 4 } & Walking & 0.519 & 0.370 \\
\cline { 2 - 4 } & Running & 1.324 & 0.548 \\
\cline { 2 - 4 } & Cycling & 0.829 & 0.661 \\
\cline { 2 - 4 } & Upstairs & 1.511 & 0.341 \\
\hline
\end{tabular}

\section{B. Results of the Mono-exponential MET Estimation} Equation

In order to improve the accuracy of MET estimation generated by multiple MET regression models, a monoexponential MET estimation equation was proposed to estimate MET during non-steady state exercise. The mean and STD of MET estimation errors for the two methods are listed in Table IV each activity. We observe that average residual error and SEE for Method (3) is smaller than for Method (2). Overall, the accuracy of Method (3) outperforms Method (2).

TABLE III. COMPARISONS OF MET ESTIMATION BETWEEN METHODS (2) AND (3)

\begin{tabular}{|c|c|c|c|}
\hline MET estimation methods & Mean errors & $\begin{array}{c}\text { STD of } \\
\text { errors }\end{array}$ & SEE \\
\hline Method (2) & 0.613 & 0.709 & 0.912 \\
\hline Method (3) & $\mathbf{0 . 5 7 5}$ & $\mathbf{0 . 6 4 5}$ & $\mathbf{0 . 8 8 8}$ \\
\hline
\end{tabular}

The SEEs of ten participants generated by Method (3) are all smaller than by Method (2), and average SEE from Method (2) is 1.129 METs, while the average SEE of Method (3) was 0.680 METs. The improvement rates for each of the ten participants were above $30 \%$. The improvement rate is expressed as follows:

Im provment rate $=\frac{\text { SEE with } \operatorname{method}(2)-S E E \text { with method }(3)}{S E E \text { with } \operatorname{method}(2)} \times 100 \%(7)$

\section{CONCLUSIONS}

A wearable activity sensor system and two MET estimation methods have been presented in this paper. The activity sensor system can be used to measure and record the acceleration signals from daily physical activities. This study first developed traditional MET regression models, including a single MET regression model and multiple MET regression models. However, due to unsatisfactory estimation results by the MET regression models for non-steady states during exercise, a mono-exponential MET estimation equation was proposed to enhance the estimation accuracy for non-steady states. From the experimental results shown, the SEEs for the multiple MET regression models and the mono-exponential MET estimation equation were 0.912 and 0.888 METs respectively, and the mean $( \pm$ STD $)$ of estimated errors were $0.613( \pm 0.709)$ and $0.575( \pm 0.645)$ METs. Therefore, the experimental results validate the effectiveness of the monoexponential MET estimation equation for the non-steady and steady states MET estimation. This result encourages us to further investigate the possibility of using our developed activity sensor system as an effective tool for long-term EE monitoring and health promotion applications.

\section{REFERENCES}

[1] I. M. Lee, H. D. Sesso, and R. S. Paffenbarger Jr, "Physical activity and coronary heart disease risk in men does the duration of exercise episodes predict risk," Circulation, vol. 102, no. 9, pp. 981-986, 2000.

[2] B. A. Calton, R. Z. Stolzenberg-Solomon, S. C. Moore, A. Schatzkin, C. Schairer, D. Albanes, and M. F. Leitzmann, "A prospective study of physical activity and the risk of pancreatic cancer among women (United States)," BioMed Central Cancer, vol. 8, no. 1, pp. 1-9, 2008.

[3] K. Y. Chen and D. R. Bassett, "The technology of accelerometrybased activity monitors: current and future," Medicine \& Science in Sports \& Exercise, vol. 37, no. 11, pp. S490-S500, 2005. 
[4] S. E. Crouter, J. R. Churilla, and D. R. Bassett Jr, "Estimating energy expenditure using accelerometers," Eur. Journal of Applied Physiology, vol. 98, no. 6, pp. 601-612, 2006.

[5] A. M. Swartz, S. J. Strath, D. R. Bassett, W. L. O’brien, G. A. King, and B. E. Ainsworth, "Estimation of energy expenditure using CSA accelerometers at hip and wrist sites," Medicine \& Science in Sports \& Exercise, vol. 32, no. 9, pp. S450-S456, 2000.

[6] P. S. Freedson, E. Melanson, and J. Sirard, "Calibration of the computer science and applications, Inc. accelerometer," Medicine \& Science in Sports \& Exercise, vol. 30, no. 5, pp. 777-781, 1998.
[7] D. Hendelman, K. C. Baggett, E. Debold, and P. Freedson, "Validity of accelerometry for the assessment of moderate intensity physical activity in the field," Medicine \& Science in Sports \& Exercise, vol. 32, no. 9, pp. S442-S449, 2000.

[8] K. L. Campbell, P. R. E. Crocker, and D. C. Mckenzie, "Field evaluation of energy expenditure in women using Tritrac accelerometers," Med. \& Science in Sports \& Exercise, vol. 34, no. 10, pp. 1667-1674, 2002.

[9] S. E. Crouter and D. R. Bassett Jr, "A new 2-regression model for the Actical accelerometer," British Journal of Sports Medicine, vol. 42, no. 3, pp. 217-224, 2008. 